\title{
Marked Improvement of Levothyroxine Malabsorption by Simply Changing the Timing of Thyroxine Ingestion in an Adult Woman With Hypothyroidism After Radioiodine Therapy for Graves' Disease
}

\author{
Yoji Miyoshi
}

\begin{abstract}
We report the case of a 37-year-old woman suffering from hypothyroidism resistant to oral levothyroxine (LT4) substitution after radioiodine therapy for Graves' disease. She was admitted to our endocrinology clinic for persistent hypothyroidism despite administration of full-dose LT4 (175 $\mu \mathrm{g} /$ day). The patient complained of severe general fatigue, leg edema, and body weight gain. Her serum free thyroxine (FT4) and thyrotropin (TSH) levels were $0.64 \mathrm{ng} /$ $\mathrm{dL}$ and $58.0 \mathrm{mIU} / \mathrm{L}$, respectively. Extensive investigations excluded disease of the small bowel, liver, and pancreas, as well as drug interactions. She also had no gastrointestinal diseases (for exmple, ulcer, inflammatory colitis). We were also able to rule out failure to take LT4 and concomitant use of other drugs. One month after changing the timing of LT4 ingestion from after breakfast to before breakfast, her serum TSH level did not decrease and her serum FT4 level increased from 0.64 to $1.02 \mathrm{ng} / \mathrm{dL}$. The symptoms of hypothyroidism remained unchanged. We then changed the timing of LT4 ingestion from morning to bedtime. One month later, her serum TSH level had normalized and signs of hypothyroidism had disappeared. This case emphasizes the clinical importance of the timing of thyroxine ingestion in not a few patients with hypothyroidism resistant to LT4, although the reason for the malabsorption in this patient remains unclear.
\end{abstract}

Keywords: Hypothyroidism; Malabsorption of thyroxine; Timing of thyroxine ingestion

\section{Introduction}

Patients with hypothyroidism are supplemented with synthetic thyroxine hormone (levothyroxine or LT4) to achieve physiological serum levels of free thyroxine (FT4). Howev-

\footnotetext{
Manuscript accepted for publication December 13, 2012

Corresponding address: Division of Endocrinology, Diabetes and Metabolism, Sayama General Clinic, 4-15-25 Irumagawa, Sayama, Saitama 350-1305, Japan. Email: y_miyoshi@ae.auone-net.jp
}

doi: http://dx.doi.org/10.4021/jem129w er, in a few cases, hypothyroidism persists and a euthyroid state cannot be achieved even after supplementation with excessive doses of oral LT4. The factors responsible for the resistance of hypothyroidism to thyroxine therapy are varied, and include variable patient adherence of LT4, various malabsorption disorders affecting LT4 absorption, and medications and food known to interfere with the intestinal absorption and metabolism of LT4 [1-3]. If the above possibilities are excluded, the timing of LT4 administration can also affect serum thyrotropin (TSH) concentrations. Bach-Huynh et al. showed that TSH levels were higher when LT4 was taken at bedtime instead of before breakfast [4]. However, Bolk et al. observed several patients whose thyroid hormone levels improved after LT4 ingestion was changed from the morning to bedtime [5]. It thus remains unclear whether the timing of LT4 ingestion has a clinically significant impact on serum TSH levels.

We describe a patient with hypothyroidism who was resistant to full-dose LT4 and whose serum TSH levels and clinical symptoms strikingly improved after simply changing the time of LT4 ingestion from morning to bedtime.

\section{Case Report}

The patient was a 37-year-old Japanese woman who presented to an outpatient endocrinology clinic with the typical symptoms of hyperthyroidism (namely, palpitations, weight loss, increased sweating). Laboratory analysis of her blood and ultrasonography of her thyroid gland led to the diagnosis of Graves' hyperthyroidism. Treatment with propylthiouracil (PTU, 3 tablets/day) was initiated in May 2009. However, her clinical features and laboratory data were not improved. After 6 months of treatment, the decision was made to inhibit thyroid function with radioactive iodine. The patient was orally administered $370 \mathrm{MBq}$ of radioactive iodine in July 2009. She subsequently developed hypothyroidism with low serum FT4 and high serum TSH levels concomitant with weight gain, general fatigue, myxedema, and muscle cramps. After 3 months of treatment with radioactive iodine, in October 2009, she began to be treated with oral LT4 after breakfast. However, there was no normalization of her serum FT4 


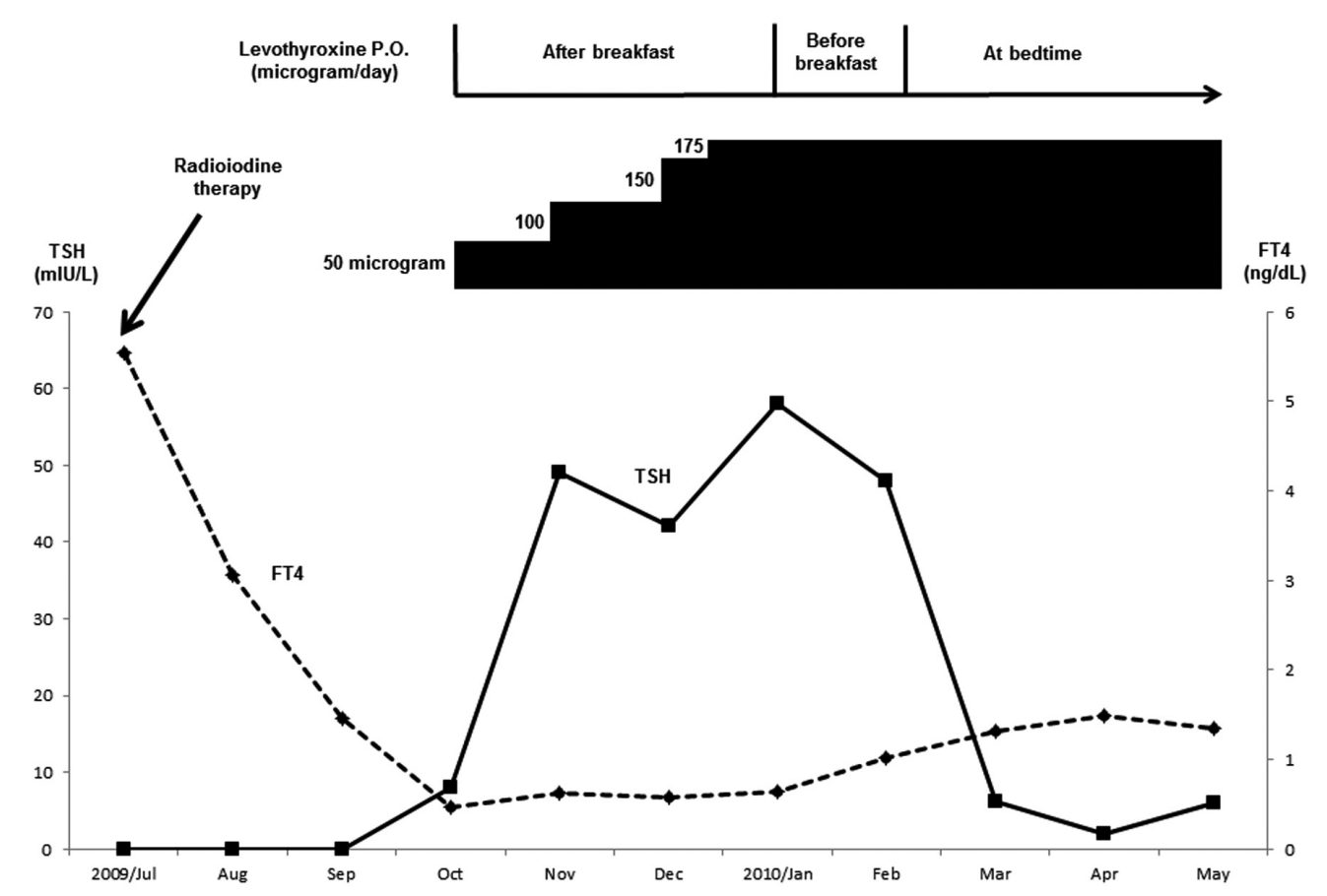

Figure 1. Clinical course. After radioactive iodine therapy was initiated in July 2009, the patient developed hypothyroidism. In October 2009, she was treated with 50 microgram of levothyroxine (LT4). Although the LT4 dose was increased to 175 microgram, her serum FT4 and TSH levels and clinical signs did not improve. In January 2010, the timing of LT4 ingestion was changed from after breakfast to before breakfast. Since no improvement was observed in her serum TSH levels, the timing of LT4 ingestion was then changed to at bedtime. One month later (in March 2010), her serum TSH level had normalized and her clinical symptoms had disappeared.

and TSH levels, even at high dosages of 175 microgram/day.

After taking 175 microgram of LT4 for 6 weeks, the patient was referred to our endocrinology clinic for further evaluation in January 2010, at which time her serum FT4 and TSH levels were $0.64 \mathrm{ng} / \mathrm{dL}$ and $58.0 \mathrm{mIU} / \mathrm{L}$, respectively. She complained of severe general fatigue and leg edema. She had no significant medical or psychiatric history in adulthood. In childhood, she had felt general fatigue and muscle weakness and she was diagnosed with myasthenia gravis (MG). However, she experienced only minor symptoms and she did not receive any treatment. At the age of 30 , she underwent a normal pregnancy and delivery without complications.

Our analyses excluded malabsorption of LT4 due to the use of other medicines or supplements. Liver and pancreatic diseases were also excluded by laboratory investigation. Additionally, the patient had no history of gastrointestinal surgery. Congestive heart failure and pregnancy were also not present. Gastrointestinal endoscopy was unremarkable. Since the patient had taken LT4 after breakfast, we recommended that she take LT4 before breakfast. Six weeks later, her serum TSH level did not decrease $(48.1 \mathrm{mIU} / \mathrm{L})$, but her serum FT4 level increased from 0.64 to $1.02 \mathrm{ng} / \mathrm{dL}$ (reference $1.00-1.70$ ) when the timing of LT4 ingestion was changed from after breakfast to before breakfast (Fig. 1).
Although we achieved normalization of her serum FT4 level to physiological levels, her serum TSH level did not improve and symptoms of hypothyroidism persisted. We subsequently changed the timing of LT4 ingestion from morning to bedtime. One month later, her serum TSH level had strikingly normalized and the clinical signs of hypothyroidism (general fatigue and muscle cramps) had disappeared (Fig. 1).

Two years later (in August 2012), she took the same dose of LT4 (175 microgram/day) at bedtime. She was healthy and her serum FT4 and TSH levels were $0.72 \mathrm{ng} / \mathrm{dL}$ and 1.74 $\mathrm{mIU} / \mathrm{L}$, respectively.

\section{Discussion}

It is rare to encounter cases of clinical and biochemical hypothyroidism that persist despite the administration of high doses of oral LT4. The mean treatment dosage of LT4 is 1.6 microgram/kg body weight/day. Enteral absorption of LT4 is approximately $70-80 \%$ of the administered dose, but this varies among patients [6]. In some cases, TSH levels may remain elevated despite treatment with high-dose LT4. In the case of refractory hypothyroidism, possible biological causes should be investigated. Factors that can inhibit the ability to maintain a desired TSH concentration include (a) 
variable patient adherence; (b) various malabsorption disorders affecting LT4 absorption; and (c) medications and food that affect LT4 absorption and metabolism [1-3]. In our case, we closely monitored our patient by remaining in contact via email and conducting follow-up visits. She clearly indicated that her intake of LT4 was as prescribed, and so we believe that her compliance with oral LT4 was good. During these observations, she reported that she was not taking any medications or supplements other than LT4. She had no significant illness except for MG, which was diagnosed in childhood. Since then, she had been doing well until she developed Graves' disease. She underwent a normal pregnancy and delivery at the age of 30 without any complications. She had no gastrointestinal, liver, or pancreatic diseases, and no congestive heart failure or current pregnancy.

Regarding the timing of LT4 ingestion, it is believed that absorption of LT4 is higher in a fasting state. Bach-Huynh et al. showed that TSH levels achieved in the fasting state were lower than TSH values under non-fasting conditions [4]. This suggests that the absorption of LT4 is optimum under fasting conditions. On the other hand, Bolk et al. showed that bedtime intake of LT4 improved thyroid hormone levels. They mentioned that the improvement may be because of better gastrointestinal bioavailability at night or reduced uptake interference by food or medications [5]. Moreover, Elliott et al. showed that thyroid function did not differ between morning and bedtime regimens [7]. It remains unclear why these results differ. It is possible that meals with different carbohydrate, protein, and fat contents have different degrees of impact on LT4 absorption and serum TSH levels. It should also be noted that these studies were performed in Western countries (USA and The Netherlands), where eating habits might be different from those in our country (Japan).

To our knowledge, there is no report describing the striking improvement of hypothyroidism by simply changing the timing of LT4 ingestion, in the literature to date. The reason for LT4 malabsorption in this patient remains unsolved. Further studies might be needed to fully understand the mechanisms for thyroxine transport and absorption.

\section{Conclusion}

We report a rare case highlighting the importance of the timing of LT4 ingestion. Considering the worldwide prescription of LT4, the timing of LT4 ingestion should be carefully considered in not a few patients with hypothyroidism resistant to LT4 supplementation.

\section{Consent}

The patient has provided informed consent for publication of this case report.

\section{Abbreviations}

LT4: levothyroxine; FT4: free thyroxine; TSH: thyrotropin.

\section{Competing Interests}

The author declares that he has no competing interests.

\section{References}

1. Morris JC. How do you approach the problem of TSH elevation in a patient on high-dose thyroid hormone replacement? Clin Endocrinol (Oxf). 2009;70(5):671-673.

2. Bell DS, Ovalle F. Use of soy protein supplement and resultant need for increased dose of levothyroxine. Endocr Pract. 2001;7(3):193-194.

3. Zamfirescu I, Carlson HE. Absorption of levothyroxine when coadministered with various calcium formulations. Thyroid. 2011;21(5):483-486.

4. Bach-Huynh TG, Nayak B, Loh J, Soldin S, Jonklaas J. Timing of levothyroxine administration affects serum thyrotropin concentration. J Clin Endocrinol Metab. 2009;94(10):3905-3912.

5. Bolk N, Visser TJ, Nijman J, Jongste IJ, Tijssen JG, Berghout A. Effects of evening vs morning levothyroxine intake: a randomized double-blind crossover trial. Arch Intern Med. 2010;170(22):1996-2003.

6. Mandel SJ, Brent GA, Larsen PR. Levothyroxine therapy in patients with thyroid disease. Ann Intern Med. 1993;119(6):492-502.

7. Elliott DP. Effect of levothyroxine administration time on serum TSH in elderly patients. Ann Pharmacother. 2001;35(5):529-532. 